\title{
Reviewer Acknowledgements for Global Journal of Health Science, Vol. 10, No. 1
}

Global Journal of Health Science wishes to acknowledge the following individuals for their assistance with peer review of manuscripts for this issue. Their help and contributions in maintaining the quality of the journal are greatly appreciated.

Global Journal of Health Science is recruiting reviewers for the journal. If you are interested in becoming a reviewer, we welcome you to join us. Please find the application form and details at http://recruitment.ccsenet.org and e-mail the completed application form to gjhs@ccsenet.org.

\section{Reviewers for Volume 10, Number 1}

Amy Clements-Cortes, University of Toronto, Canada

António Calha, Polytechnic Institute of Portalegre, Portugal

Domitila Augusta Huber, Federal University of Santa Catarina, Brazil

Evanthia Sakellari, Technological Educational Institute of Athens, Greece

Fengsong Gao, The University of Queensland, Australia

Gabriele Messina, University of Siena, Italy

Hadii M Mamudu, East Tennessee State University, United States

Jaime Hinzpeter, Clinical Hospital University of Chile, Chile

Jan Chrastina, Palacký University, Czech Republic

Jason Tsai, Lincoln College, United Kingdom

José Joaquín Mira, Universidad Miguel Hernández, Spain

Jose R Cordon, Universidad de Cadiz (Spain); SASEMAR(M. Fomento-Spain)-Spanish Goverment, Spain

Kartheek R Balapala, University Tunku Abdul Rahman, Malaysia

Loray Daws, British Columbia Masterson Institute, Canada

Matteo Vitali, Hygiene at Sapienza University, Italy

Meng Zhao, Texas A\&M University at Corpus Christi, United States

Montarat Thavorncharoensap, 1. Mahidol University 2. Health Intervention and Technology Assessment Program (HITAP), Ministry of Public Health, Thailand

Nant Thin Thin Hmwe, University of Newcastle, Australia

Pedram Iranmanesh, Dentist, Independent Researcher, Iran, Islamic Republic of

Pi-Ming Yeh, Missouri Western State University, United States

Raymond Jagessar, University of Guyana, Guyana

Roger Ho, National University of Singapore, Singapore

Ru-Jeng Teng, Medical College of Wisconsin, United States

Santha James, Australian Catholic University, Australia

Sara Melo, Queen's University Belfast, United Kingdom

Satoshi Horiuchi, Iwate Prefectural University, Japan

Soon Soo Hoo, Royal North Shore Hospital. Sydney. Australia, Australia

Srikrishna Sulgodu Ramachandra, Public Health Foundation of India, India

Steven Hoffman, University of Texas at San Antonio, United States

Tamilselvi Rajendran, Sethu Institute of Technology, India

Tan Ching Siang, Universiti Sciences Malaysia, Malaysia

Thammanard Charernboon, Thammasat University, Thailand

Trisha Dunning, Deakin University and Barwon Health, Australia 